\title{
A web-based application for automated quantification of chemical gradients induced in microfluidic devices
}

\author{
M. Cóndor ${ }^{1}$, T. Rüberg ${ }^{2}$, C. Borau ${ }^{1}$, J. Piles ${ }^{3}$, J.M. García-Aznar ${ }^{1}$ \\ ${ }^{1}$ Aragón Institute of Engineering Research (I3A), Department of Mechanical Engineering, University of \\ Zaragoza, Zaragoza, Spain \\ ${ }^{2}$ TailSiT GmbH, Graz, Austria \\ ${ }^{3}$ Max Planck Institute for Intelligent Systems, Tübingen, Germany
}

Corresponding author: J.M. García-Aznar, imgaraz@unizar.es, Tel. +34-976762796

Maria de Luna 3, Campus Río Ebro, Edificio “Agustín de Betancourt”, 50018, Zaragoza, (Spain) 


\section{Abstract}

Background and objective: Advances in microfabrication have allowed the development and popularization of microfluidic devices, which are powerful tools to recreate three-dimensional (3-D) biologically relevant in vitro models. These microenvironments are usually generated by using hydrogels and induced chemical gradients. Going further, computational models enable, after validation, the simulation of such conditions without the necessity of real experiments, thus saving costs and time. In this work we present a web-based application that allows, based on a previous numerical model, the assessment of different chemical gradients induced within a 3-D extracellular matrix.

Methods: This application enables the estimation of the spatio-temporal chemical distribution inside microfluidic devices, by defining a first set of parameters characterizing the chip geometry, and a second set characterizing the diffusion properties of the hydrogel-based matrix. The simulated chemical concentration profiles generated within a synthetic hydrogel are calculated remotely on a server and returned to the website in less than 3 minutes, thus offering a quick automatic quantification to any user. To ensure the dayto-day applicability, user requirements were investigated prior to tool development, pre-selecting some of the most common geometries. The tool is freely available online, after user registration, on http://m2be.unizar.es/insilico cell under the software tab.

Results: Four different microfluidic device geometries were defined to study the dependence of the geometrical parameters onto the gradient formation processes. The numerical predictions demonstrate that growth factor diffusion within 3-D matrices strongly depends not only on the physics of diffusion, but also on the geometrical parameters that characterizes these complex devices. Additionally, the effect of the combination of different hydrogels inside a microfluidic device was studied.

Conclusions: The automatization of microfluidic device geometries generation provide a powerful tool which facilitates to any user the possibility to automatically create its own microfluidic device, greatly reducing the experimental validation processes and advancing in the understanding of in-vitro 3-D cell responses without the necessity of using commercial software or performing real testing experiments.

\section{Keywords}

Growth factor transport; Finite element simulation; Microfluidic devices; Web-based application.

\section{Introduction}

The study of cell motility plays a key role in the understanding of numerous biological processes such as embryogenesis, immune defense, wound healing or the development of certain diseases such as cancer invasion or metastasis [1-4]. Several growth factors have been identified to stimulate cell motility, and the signaling mechanisms that mediate this induced cell movement have been thoroughly studied [5-7]. In particular, individual directional cell migration (which encompasses chemosensing, polarization and locomotion) is distinguished as a function of the signaling pathway [8]. Broadly speaking, depending on the chemical stimulus that originates the movement, two migration types are defined: chemotaxis, if cells migrate towards a soluble chemoattractant; or haptotaxis, if a growth factor (GF) bound to the extracellular matrix (ECM) influences cell motility $[9,10]$. Hence, the study of a GF diffusion behavior within the ECM, as well as the associated adhesion and degradation processes, is critical for the understanding of the chemotactic and haptotactic gradients generated by these biomolecules, which directly affect cellular behavior.

In recent years, numerous research studies have focused on recreating in vitro 3D cell cultures capable to replicate many aspects of in vivo cell microenvironments [11]. The main advantages that in vitro assays present compared to in vivo studies are their relatively easy handling and high reproducibility. Furthermore, recent advances in microfluidic technologies have enabled the development of new generation microfluidic 
devices, allowing the production of physiologically relevant in vitro cellular microenvironments, by including biomimetic hydrogels and the generation of controlled chemical gradients $[12,13]$. These models furthermore allow high quality visualization and quantification of cell responses in vitro, and in particular the study of GF behavior within 3-D matrices, as well as the associated adhesion and degradation processes [14$16]$.

Quantification of growth factor signaling in 3-D matrices has greatly increased the understanding of directional cell migration processes, addressing several issues: the degree to which these factors can concentrate within the 3-D matrices, such as collagen or fibrin hydrogels and, consequently, act as signaling molecules driving cell motility [14]. Nevertheless, there are still several unresolved issues, such as the study of the dependence of the geometrical/design parameters of these devices on the gradient formation processes or the development of a powerful computational tool that allow an automatic quantification of growth factor signaling within a biopolymer network.

Thus, in this work we present a finite element model based on a reaction-diffusion transport model accompanied by a web-based application, to automatically calculate the distribution of different growth factors, across hydrogel-based matrices (specifically collagen type I and fibrin). This application yields an accurate prediction, based on previous experimental results, where diffusion and binding phenomena can be estimated within the microdevice. To that end, quantitative ELISA-assays for growth factor concentrations measurement were performed and the behavior of die dextran diffusion gradient was studied, validating therefore the proposed numerical model [14].

Among many others GFs, platelet derived growth factor-BB (PDGF-BB) and transforming growth factor$\beta_{1}\left(\right.$ TGF- $\left.\beta_{1}\right)$ have been selected to perform the sensibility/parametrical analysis across collagen and fibrin hydrogels. These GFs have received increasing attention in the last years due to their diverse biological effects. For instance, PDGF-BB is a pro-migratory factor that plays a key role in the early stage of wound healing, whereas TGF- $\beta_{1}$ stimulates fibroblasts differentiation into contractile myofibroblasts. Furthermore, the binding capability of these GFs to different ECM components exerting their biological activity has been reported [17-19]. Due to the considerable similarity encountered between synthetic hydrogels and the native extracellular matrix, synthetic hydrogels can serve as model systems for studying the transport of a soluble molecule and the formation of chemical gradients in biological systems. The hydrated polymer chains of synthetic hydrogels promote a slow movement of the solute as well as the macromolecules existing within the ECM, thus enhancing gradient formation [20, 21]. In this work, the diffusion of both GFs was studied on two different hydrogel-based matrices, namely collagen type I and fibrin. Collagen I is one of the major components of the connective tissue, up to $30 \%$ of total human protein; and fibrin is an essential component of healing and angiogenic processes [22-24].

This work has focused on the parameterization of four different base designs of microfluidic devices to analyze the diffusive behavior of a growth factor within a fibrillar network, thereby facilitating the main parameters that characterize the gradient formation process inside these microdevices. The finite element analysis of the numerical model proposed has been performed with an unstructured finite element mesh, based on linear triangle elements and an Euler backward time integration scheme [25]. The results demonstrate that growth factor diffusion within 3-D matrices clearly depends not only on the physics of diffusion, but also on the geometrical parameters that characterizes these complex devices.

Moreover, in this work we present a novel web-based application which facilitates to any user an automatic estimation of growth factor diffusion within 3-dimensional matrices. This application allows the user to define the geometrical parameters that characterize each chip design (hydrogel height and width or number of posts among others); as well as the diffusion-related parameters: the type of growth factor (PDGF-BB or TGF- $\beta_{1}$ ), the initial GF concentration, the matrix (collagen of fibrin) and the diffusion time to 
simulate. This platform therefore facilitates any user the possibility to customize its own microfluidic device and automatically simulate the chemical conditions that would regulate in vitro 3D cell responses.

\section{Methods}

\section{Numerical model of GF transport within synthetic hydrogels}

During the transport of a biomolecule within a synthetic hydrogel, these can interact in distinct ways with the fibrillar network: as a purely diffusive factor in the soluble fluid or bound to the matrix proteins. Moreover, the degradation of the factor takes place at the same time as a result of their interaction with other biomolecules $[18,26,27]$.

To determine the concentration gradients stablished inside a synthetic hydrogel, we use a reactiondiffusion model where the rate of change of concentration depends on a diffusive term (which explains the movement of the factor) and a reaction term that encorporates the degradation and the binding of the factor to the fibrillar network:

$$
\frac{\partial C(x, t)}{\partial t}=D_{i} \nabla^{2} C(x, t)+R_{i} \quad i=1,2
$$

where index $i$ refers to the microfluidic domain where equation 1 is evaluated, $C$ is the concentration of the factor, $D_{i}$ is the effective diffusion coefficient and $R_{i}$ the reactive term. We consider that that binding phenomena can only take place in the central domain of the device where the hydrogel is situated (see Fig. 1). Therefore, the reactive term can be defined as a function of the microfluidic device domain:

$$
R_{i}= \begin{cases}-k_{d} C(x, t) & \text { if } i=1 \text { (lateral channel) } \\ -k_{b} C(x, t) & \text { if } i=2 \text { (central channel) }\end{cases}
$$

where $k_{d}$ and $k_{b}$ are the degradation and binding rates respectively. Both coefficients were numerically estimated for each particular case of study, to reduce the average difference on concentrations quantifications obtained experimentally [14].

The transport of a soluble biomolecule within a synthetic hydrogel depends not only on the solute properties but also onto the fibrillar network ones. Then, standard and effective diffusion coefficients were computed by means of Stokes-Einstein equation and Ogston-Kim formulation respectively [28-30]:

$$
\begin{aligned}
& D_{\infty}=\frac{K_{B} T}{6 \pi \eta r} \\
& D_{i}=D_{\infty} e^{-\left[\sqrt{v_{v}}\left(1+\frac{r}{r_{t}}\right)\right]}
\end{aligned}
$$

where $K_{B}$ is the Boltzmann constant, $T$ the absolute temperature, $\eta$ the viscosity of the fluid, $v_{v}$ the void ratio of the matrix, $r$ the radius of the biomolecule to diffuse and $r_{f}$ the fiber radius of the fibrillar network.

For further information about the experimental quantification of GF concentration and degradation as well as diffusion coefficients estimation and matrix measurement properties, see our previous works [14, 31]. 


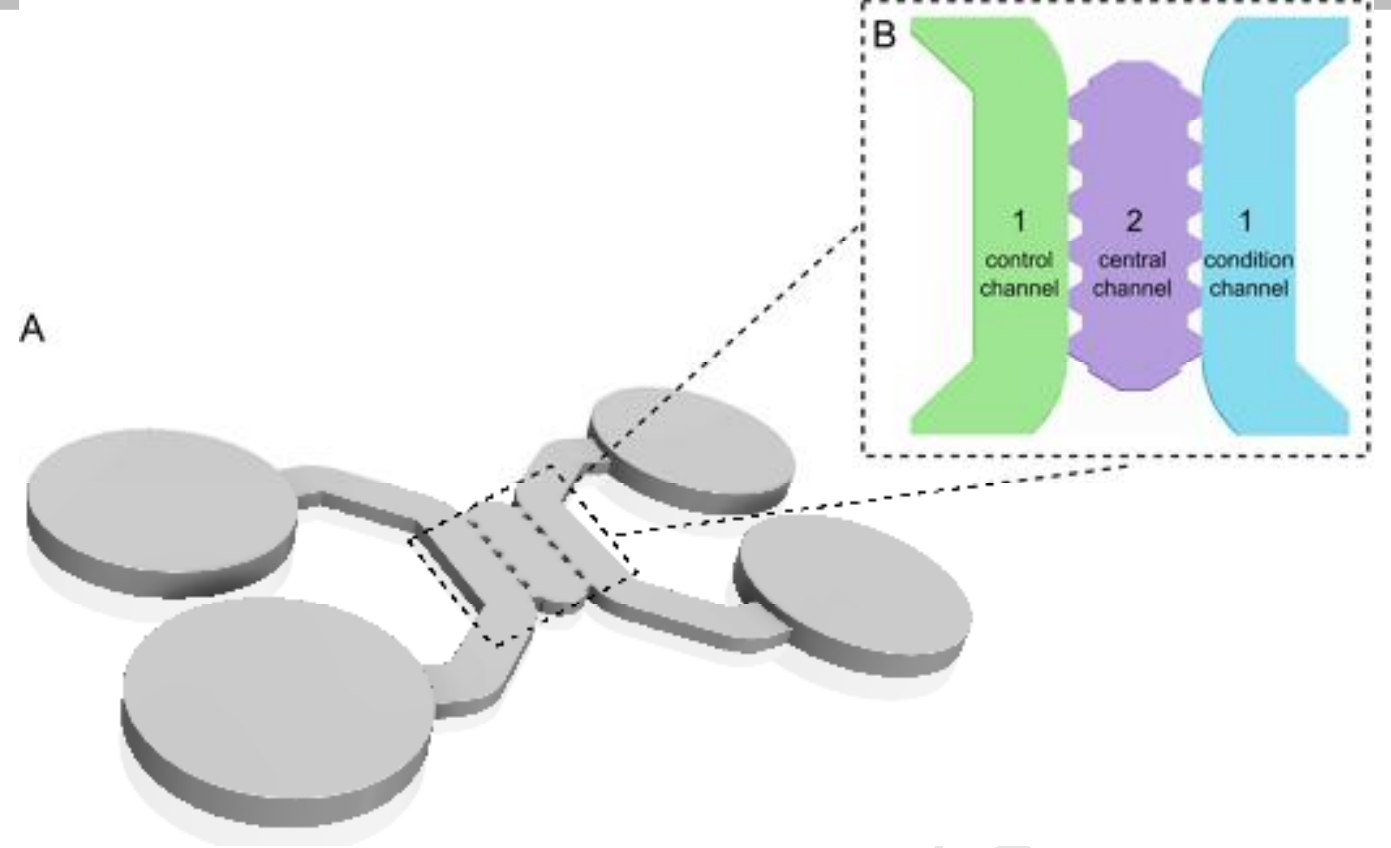

Figure 1. Geometry of a microfluidic-based device. A) Three-dimensional view and B) detail of the central area of the device, specifying the different domains where the Eq. 1 is evaluated. Domain 1 corresponds with the control and condition channels (used to introduce a GF and induce a chemical gradient) and domain 2 corresponds with the central channel of the device where the hydrogel and cells are situated during the experimental setup.

\section{Microfluidic device geometry parameterization}

An automatic generation of microfluidic device geometries was implemented to facilitate an automatic quantification of a diffusion case inside any microfluidic device geometry. To study how the different geometrical parameters that characterize microfluidic devices affect diffusion gradients on in vitro 3D cell cultures, four base geometries of microfluidic devices were parameterized. These models are currently used in real experimental assays for studying different biological processes, such as angiogenesis [32], directional cell migration [14], wound healing [33], or even for drug screening experiments. In the following text, these 4 devices are referred as chip-1, chip-2, chip-3 and chip- 4 attending to the nomenclature showed in Fig. 2B-E.

These microdevices are basically composed by a central channel, in which the hydrogel or the matrix is located; and two lateral channels (condition and control channels) on both sides of the central channel and in direct connection with the hydrogel, ensuring therefore the diffusion and hydration of the hydrogel and the cells during the experiments development (see Fig. 1). Due to their geometrical features, the most versatile device is possibly chip-1, which is used for studying 3D directional cell migration processes. Also, chip 4 is worth highlighting because of its special design, composed by 2 different central compartments where two different hydrogels can be simultaneously introduced, allowing therefore studying wound healing processes and the interface region between two different scaffolds or hydrogels (e.g. collagen and fibrin hydrogels) [33].

The generation of concentration gradients inside a microfluidic device is highly dependent on the geometry of the gradient formation region, which is basically defined by the central area of the device where the hydrogel is situated, and conditioned by both lateral channels (control and condition channel). The diffusion gradient generated in the central channel of the device depends on the volume enclosed within it, being defined mainly by the hydrogel scaffold height and width $\left(H_{g e l}\right.$ and $\left.W_{\text {gell }}\right)$. In addition to these two parameters, this region is also determined by the area enclosed by the posts as well as the post shape, 
mainly affecting the flow regime generated and to the flow speed. For studying the post role, two different column/post geometries have been implemented: trapezoidal (chip-1 and chip-2) and rectangular (chip-3 and chip-4). Moreover, for a given hydrogel height, the diffusion gradient will be conditioned by the height and the number of posts situated inside the device $\left(H_{p}\right.$ and $N_{p}$ respectively) (see Fig. 2A).

Moreover, for in-vitro cell migration experiments, the spatial distribution of the posts will limit the movement of cells between channels, being limited therefore by the available inter-space between the posts. If the separation between posts is smaller than the average cell size, cells will not be able to pass through the posts during experiments. The average size of eukaryotic cells usually ranges from $10-100 \mu \mathrm{m}$ [34]. Thus, the minimum distance between two posts $\left(H_{\min }\right)$ was fixed equal to $50 \mu \mathrm{m}$ and therefore cannot be modified by the user (see Fig. 2A).

A

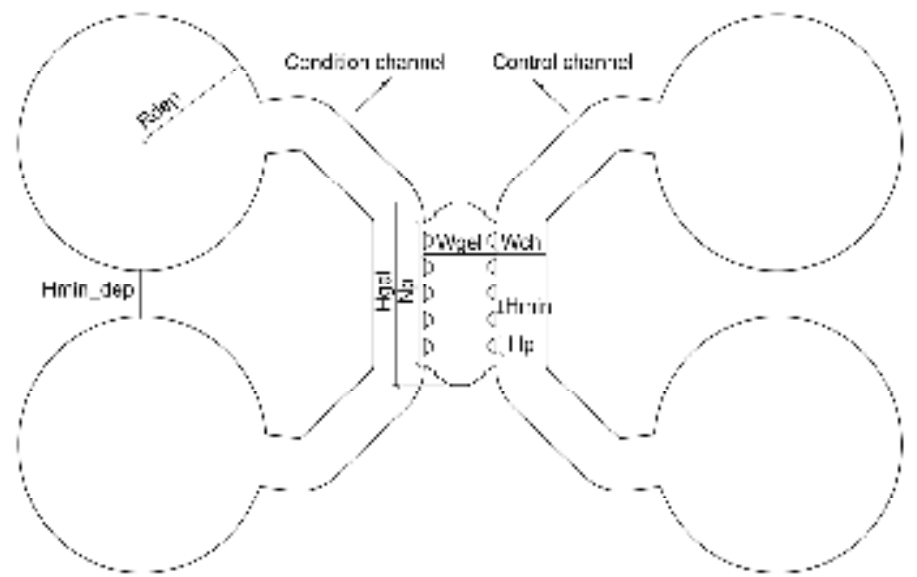

C

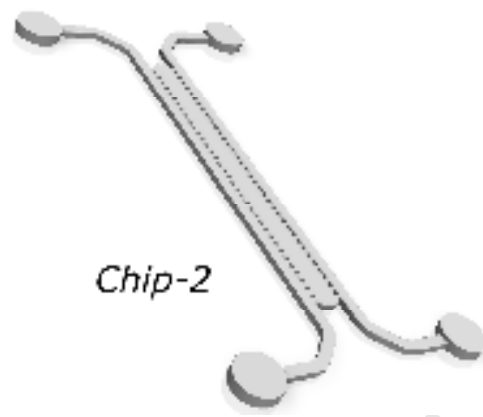

B
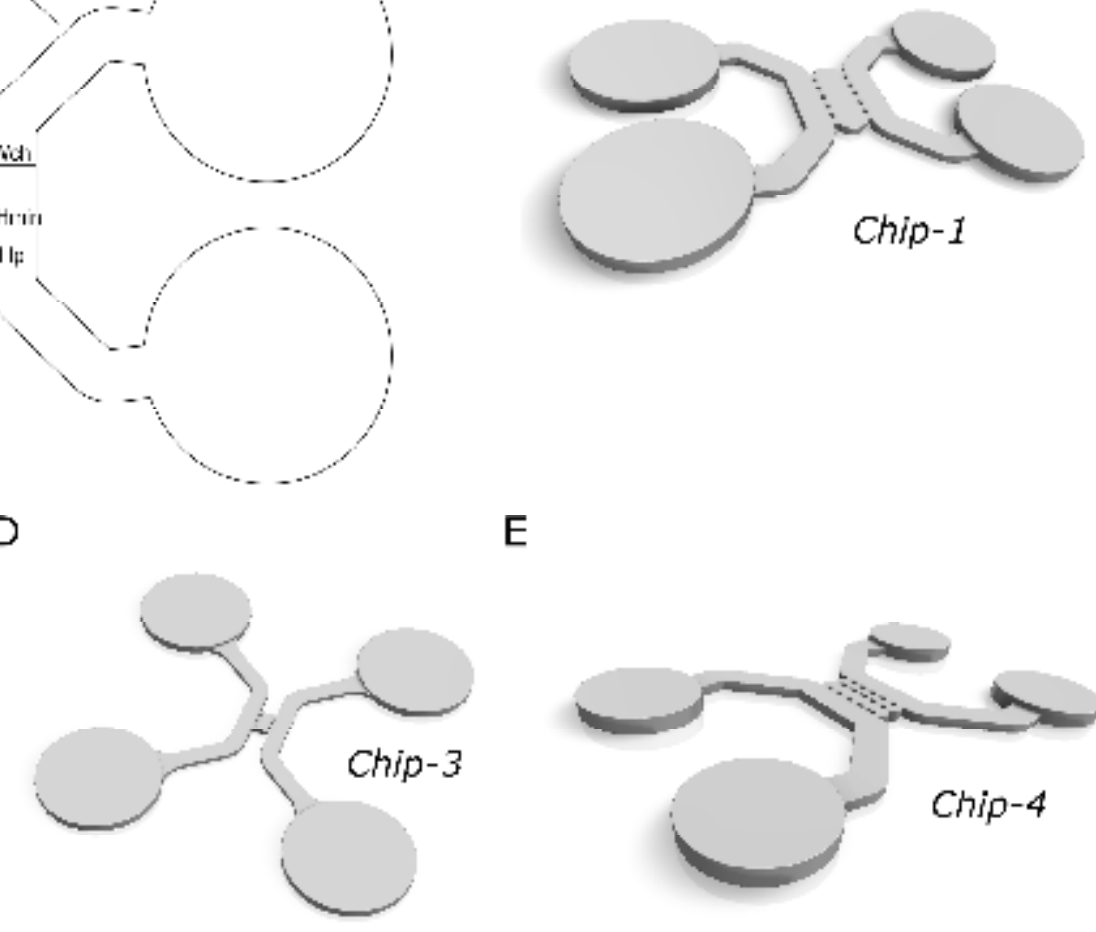

$\mathrm{E}$

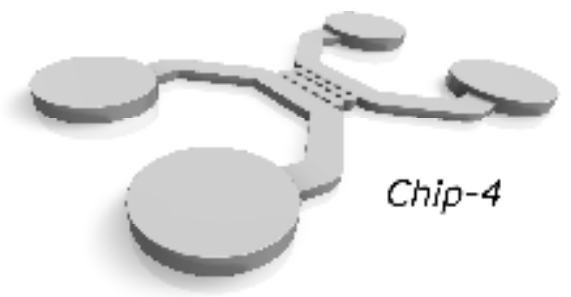

Figure 2. Parameterization of a microfluidic-based device. A) Parameters defining automatic microfluidic device geometry. $R_{\text {dep }}$ stands for the deposit radius (unnecessary for the simulations), $H_{\text {gel }}$ and $W_{\text {gel }}$ are the hydrogel height and width respectively, $W_{c h}$ is the lateral channels weight, $H_{p}$ and $N_{p}$ are the post height and number of posts, and $H_{\min }$ and $H_{\text {min_dep }}$ are the minimal height between posts and deposits respectively. The four device geometries used for the parametric study: B) Chip-1 which currently is used for studying directional cell migration, C) chip-2 which is employed for studying angiogenesis, D) chip-3 which is currently used for following-up and monitoring of drugs and E) chip-4 which is the double channel device and is used for studying wound healing processes. The parameter values that define the four chip geometries are all reported in Table 1. 


\section{Table 1}

\section{ACCEPTED MANUSCRIPT}

Main geometrical parameters that define chips designs showed in figure 2 . All parameter values are in $\mathrm{mm}$.

\begin{tabular}{ccccc}
\hline Parameter & Chip 1 & Chip 2 & Chip 3 & Chip 4 \\
\hline$H_{g e l}$ & 3.2 & 15.22 & 1.44 & 3 \\
$W_{g e l}$ & 1.3 & 1.3 & 1 & 1.5 (0.75 for each gel) \\
$W_{c h}$ & 1 & 0.5 & 1 & 0.9 \\
$H_{p}$ & 0.3 & 0.3 & 0.16 & 0.25 \\
$N_{p}$ & 5 & 36 & 3 & 5 \\
\hline
\end{tabular}

With regard to the lateral channels, it is assumed that they have the same width $\left(W_{c h}\right)$ and that there are no differences between control and condition channels radii, thus maintaining the symmetry of the microfluidic device. To avoid deposit overlapping during the automatic microfluidic device geometry generation, a minimum distance between deposits was stablished $\left(H_{\text {min_dep }}\right)$. This distance is fixed, equal to $50 \mu \mathrm{m}$ and not modifiable by the user (see Fig. 2A).

Finally, to extend the two-dimensional (2-D) geometry parameterization to a three-dimensional (3-D) one, the depth of the hydrogel $\left(D_{\text {chip }}\right)$ which is constant throughout the entire domain of the device, must be set for the simulations. For all devices the depth is fixed to $120 \mu \mathrm{m}$ and not modifiable by the user.

Once all parameters are set, the microfluidic device geometry is generated with a custom python code. Through the parameterization of these microdevices, any device can be automatically generated based on six input parameters $\left(H_{g e l}, W_{g e l}, W_{c h}, H_{p}, N_{p}\right.$ and $\left.R_{\text {dep }}\right)$, without losing the model scale, allowing a semiautomatic generation of microfluidic devices, and therefore reducing design times and facilitating experimental validation processes as well as parameter selection.

\section{D Finite element model of a microfluidic device}

The diffusion phenomena inside a microfluidic device is essentially planar, therefore we employed a 2D finite element model based on an automatically generated mesh and an Euler backward time integration scheme. The finite element mesh is automatically generated with the mesh generator GMSH [35] and the calculation it is solved with a finite element method using the custom-made library inSilico [36].

The mesh was constructed using linear triangular elements with an automatic adaptive/flexible element size varying between 100 to $400 \mu \mathrm{m}$ based on a Delaunay triangulation. The element size was inside the asymptotic region of convergence and represented a good trade-off between numerical accuracy and computational cost for all cases. Three different mesh domains were defined (Fig. 3) (condition channel: red area, central domain: green area and control channel: blue area) to obtain a better adaptive element size on each domain and no distorted elements. Moreover, a locally mesh refinement was performed in the regions of interest, such as the central domain of the device or in the interface between the lateral channels and the central domain. 

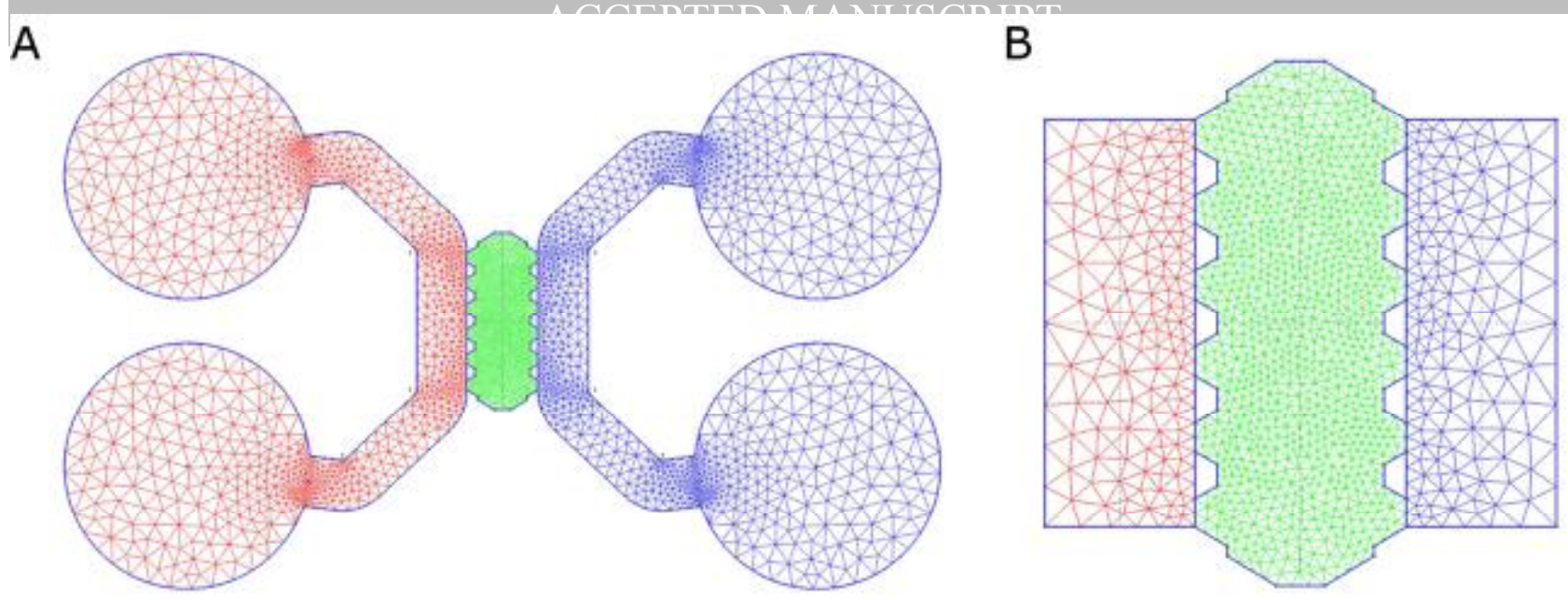

Figure 3. Finite element model of a microfluidic-based device. A) Automatic FE mesh of chip-1 geometry. B) Automatic FE mesh of the simplified chip-1 geometry.

To avoid unnecessary calculations and improve the time response to the user, the geometry was simplified by pruning the load channels. Doing this we are assuming that the chemical factor diffuses homogeneously throughout the condition channel of the device and the growth factor concentration at the initial time is constant in both lateral channels. The simplified finite element model (FEM) of chip-1 used for diffusion simulations is shown in Fig. 3B and consists of a total of 1335 vertices and 2762 triangular elements. Thus, for the simplified model only 5 input parameters will be actually necessary to automatically reconstruct the device geometry: the hydrogel scaffold height and width $\left(H_{g e l}\right.$ and $\left.W_{g e l}\right)$, the lateral channels width $\left(W_{c h}\right)$ and the post height and number of posts $\left(H_{p}\right.$ and $\left.N_{p}\right)$.

The simulation backend is based on a standard FEM formulation with Lagrange-type elements [37]. At the spatial boundaries homogeneous natural boundary conditions are used. As initial condition, a non-zero concentration was prescribed in the control channel, based on which the time-stepping started. For reasons of robustness, we employed a simple Euler backward method which is unconditionally stable [37]. Due to the small system size, we chose a Cholesky decomposition in order to solve the linear equations in each time step.

\section{Web application for the numerical model proposed: Insilico-cell}

A novel web-based application was developed for the numerical model proposed, allowing to any user the calculation of the concentration gradients stablished inside a microfluidic-based chip (see Fig. 4). To do so, a PHP code was inserted into the website of the Insilico-cell project. This code reads the parameters from the user input and sends them to a private cluster which builds the geometry, performs the calculations and returns the computed results to the web portal screen in approximately 3 minutes. To avoid cluster overflow due to bot action, all users must create an account to be able to freely use the web application here presented.

The computational requirements involved make a local calculation in the client infeasible. Even a serverside solution would put too much of a strain on server, possibly impacting the operation of the website itself, and hence causing a poor user experience. A cluster solution was thus developed where the website acts as a front-end that submits jobs to a remote computing resource using the HTCondor queueing management system [38]. 
The website will wait until the job has been processed, will then retrieve the results from the cluster, and will finally show them. This whole process is done in a way totally transparent to the user, which offers greater flexibility to upgrade the computing power according to the demand of the service. Furthermore, the computing resources used need not be exclusively dedicated to this task, since a job priority system allows to run jobs coming from the website (which are therefore much more sensitive to delays in their completion) before background ones.

A

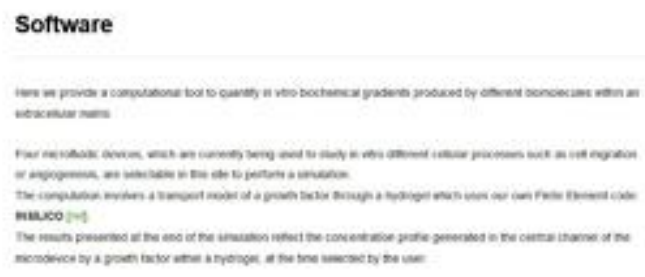

Phase seloct one of the chip models below to perform the calculation:

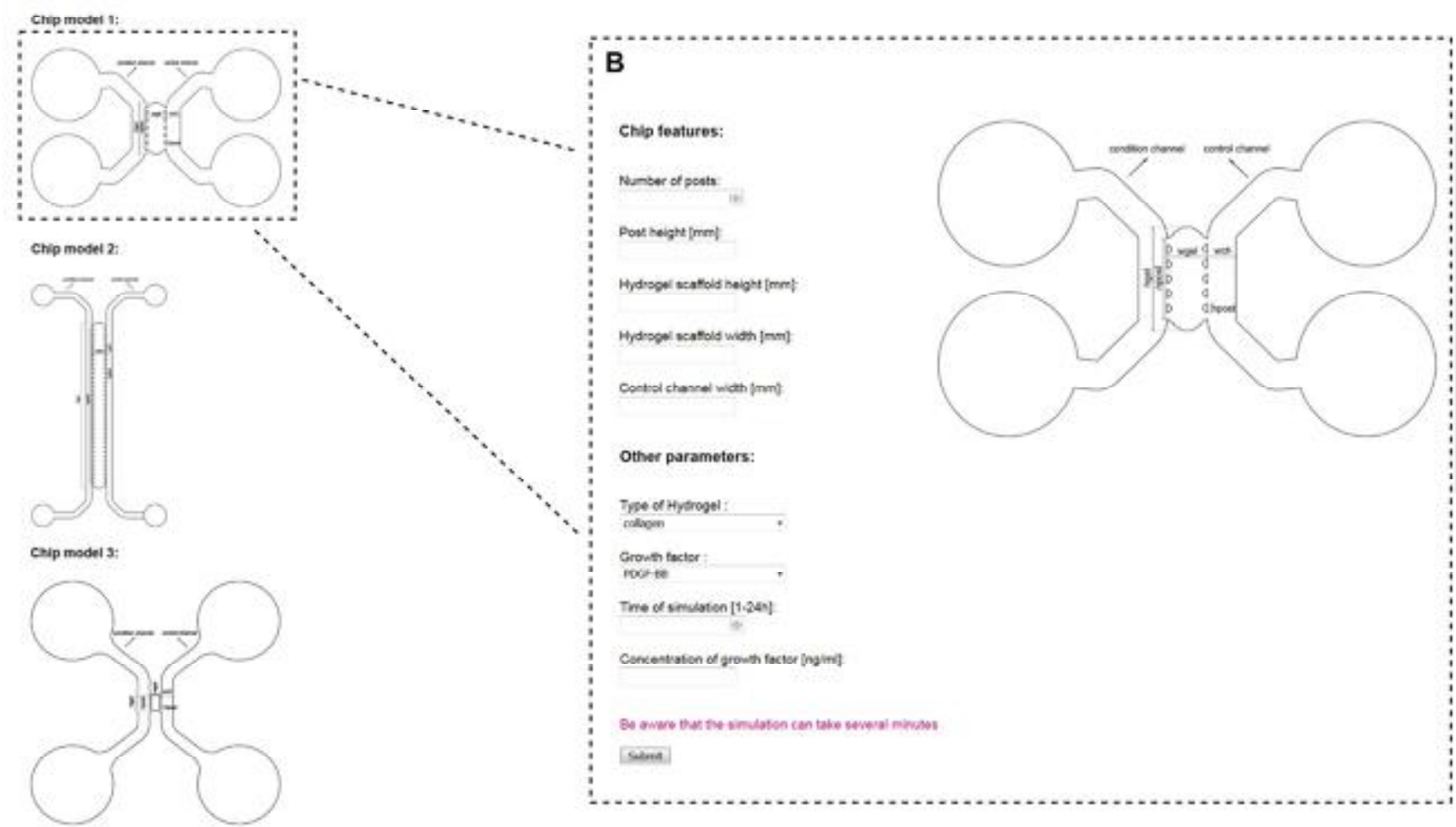

CNip model 4:

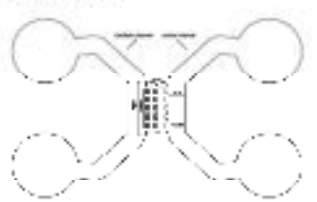

Figure 4. Screenshot of the web-based application interface. A) The four different models of microfluidic devices are presented as clickable images. B) Parameter input-window is opened after selecting any device (chip-1 on the image), where the user may select the input parameters that define the chip geometry and the calculation parameters. 
The application allows users to simulate a diffusion case choosing among the 4 different base geometries previously presented (chip-1, chip-2, chip-3 and chip-4). Once the user has selected the device geometry, the corresponding parameters-input window is opened (see Fig. 4B). Firstly, the user must set the 5 parameters defining the simplified device geometry: the hydrogel scaffold height and width $\left(H_{g e l}\right.$ and $\left.W_{\text {gel }}\right)$, the lateral channels width $\left(W_{c h}\right)$, the post height and the number of posts $\left(H_{p}\right.$ and $\left.N_{p}\right)$. Subsequently, the user must define the diffusion case defining: the growth factor (PDGF-BB or TGF- $\beta_{1}$ ), the initial growth factor concentration (any value in $\mathrm{ng} / \mathrm{ml}$ ), the hydrogel or scaffold (collagen of fibrin) and the diffusion time to simulate (up to 24 hours, see Fig. 4B). These are all the possible cases/combinations that can be currently simulated which correspond with the ones experimentally validated so far.

\section{Results}

\section{Numerical predictions of GF transport: 4 different geometries}

To evaluate the predictive capacity of the theoretical/numerical model, die dextran diffusion gradient was simulated within chip-1. Moreover, four different cases were previously experimentally validated: 24 hours of diffusion of PDGF-BB and TGF- $\beta_{1}$ in collagen and fibrin. For all cases, the numerical simulations fairly accurately predicted the experimental concentrations obtained with the ELISA assays. For more information about the experimental quantification of GF concentration and degradation as well as the numerical model validation, see a previous published work [14].

To determine whether or not there are significant differences between diffusion gradients on different models of microfluidic devices, as a first approach, the same diffusion case was simulated for the four different base models of microfluidic devices presented before. To that end, the diffusion behavior of PDGFBB growth factor on collagen gels was studied. The initial concentration of PDGF-BB applied was 50ng/ml in all cases and the total simulated time was 24 hours. The radius of this particle was estimated from Protein Data Bank to be $4.510^{-9} \mathrm{~m}$, which according to equation 3 corresponds to a standard diffusion coefficient of $1.2610^{-11} \mathrm{~m}^{2} / \mathrm{s}$, which in turn leads to an effective diffusion coefficient of PDGF-BB in collagen of $0.7710^{-}$ ${ }^{11} \mathrm{~m}^{2} / \mathrm{s}$. The collagen hydrogel characterization was previously performed with confocal reflection imaging and collagen fiber radius was estimated to be $79.51 \pm 33.16 \mathrm{~nm}$ (mean $\pm \mathrm{se}$ ) and the collagen void ratio to be $80.15 \pm 1.82 \%$ (mean $\pm \mathrm{se}$ ). The degradation and binding rates used in all simulations are $310^{-6} \mathrm{~s}^{-1}$ and $110^{-5} \mathrm{~s}^{-}$ ${ }^{1}$ respectively, both experimentally adjusted [14]. In the case of chip-4, the double channel device, a double collagen type I hydrogel was simulated.

A

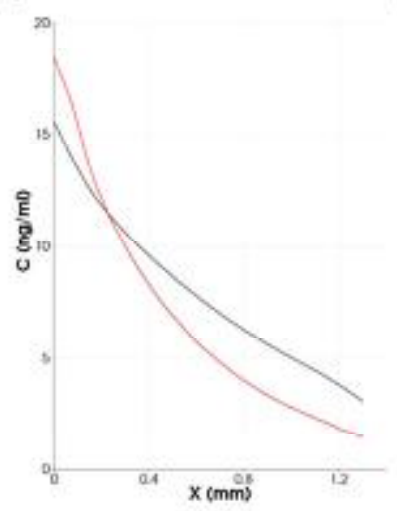

B

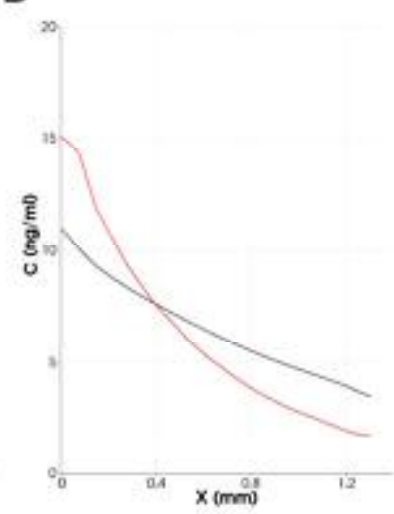

C

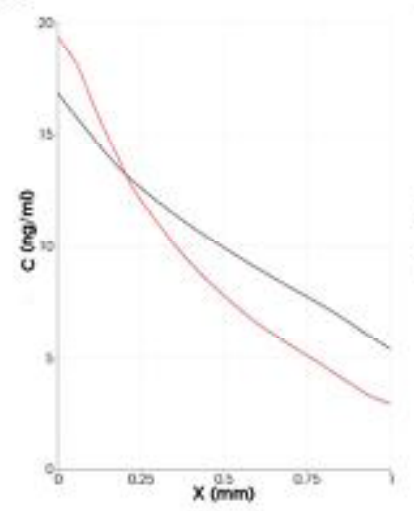

D

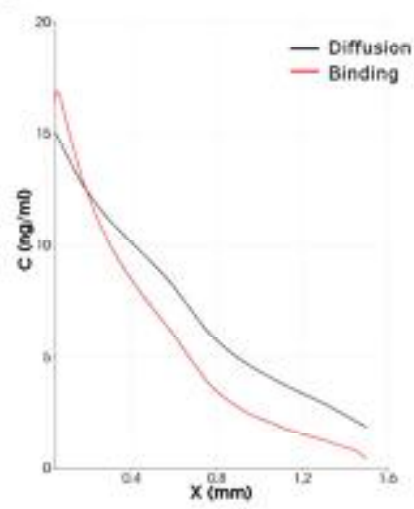

Figure 5. Numerical predictions of PDGF-BB diffusion within a collagen type I hydrogel for different microfluidic device geometries. Diffusion and binding profiles obtained after 24 hours of diffusion of PDGF-BB in the central domain of: A) chip-1, B) chip-2, C) chip-3 and D) chip-4. 


\section{ACCEPTED MANUSCRIPT}

Figure 5 shows model predictions of diffusion and binding profiles generated in the hydrogel domain or in the central channel of the device, for the different models of microfluidic devices after 24 hours of diffusion of PDGF-BB. Model predictions show different diffusion and binding profiles on each device, for equal initial concentration of PDGF-BB. As expected, these data reveal that diffusion gradients are highly dependent on the device geometric parameters and not only the diffusion-related ones. Thus, it becomes necessary to perform an intensive parametric analysis to stablish which ones are particularly relevant.

\section{Multiparametric analysis of gradient formation within a synthetic collagen-based hydrogel}

The gradient formation region of these microdevices was initially designed to generate quasi-linear concentration profiles inside them. However, as stated before, geometrical parameters have a strong impact on the concentration profiles generated inside them. To define the main parameters that characterize the gradient formation processes within a synthetic hydrogel, a multiparametric analysis of the gradient formation region was performed.

To understand the dependence of geometric parameters of microfluidic devices onto the gradient formation processes, 6 different parameters were studied: the hydrogel scaffold height and width $\left(H_{g e l}\right.$ and $\left.W_{g e l}\right)$, the lateral channels width $\left(W_{c h}\right)$, the post height $\left(H_{p}\right)$, the number of posts $\left(N_{p}\right)$ and the type or shape of posts (rectangular and trapezoidal). The simulated case was the diffusion of PDGF-BB growth factor within a collagen hydrogel and the total time simulated was 24 hours. The reference values for chip- 1 device were: $H_{g e l}=3.2, W_{g e l}=1.3, W_{c h}=1, H_{p}=0.3, N_{p}=5$ and trapezoidal posts (all values in $\mathrm{mm}$ ). Figure 6 shows the corresponding results of every case indicating the varied parameter. Degradation and binding rates are the same as previously, namely $3 \mathrm{e}^{-6}$ and $1 \mathrm{e}^{-5} \mathrm{~s}^{-1}$ respectively. The standard and effective diffusion coefficient were also maintained $\left(1.2610^{-11} \mathrm{~m}^{2} / \mathrm{s}\right.$ and $0.7710^{-11} \mathrm{~m}^{2} / \mathrm{s}$ respectively).

Some parameters such as the hydrogel height and width $\left(H_{g e l}\right.$ and $\left.W_{g e l}\right)$, the lateral channels width $\left(W_{c h}\right)$ or the posts height $\left(H_{p}\right)$ presented strong effects on the diffusion and binding profiles generated in the central domain of the device (see Fig. 6A-H), whereas the post shape repercussion was negligible. Differences on concentration for condition and control channels with respect to the reference case (chip-1 with the reference parameter values) were also quantified. Changes on hydrogel width generated differences of up to $21 \%$ on condition channel concentration and of about $24 \%$ on control channel concentrations (Fig. 6C-D respectively); On the other hand, changes on post height showed lower differences of $6 \%$ on condition channel concentration and of $13 \%$ on control channel concentrations (Fig. 6G-H respectively). 

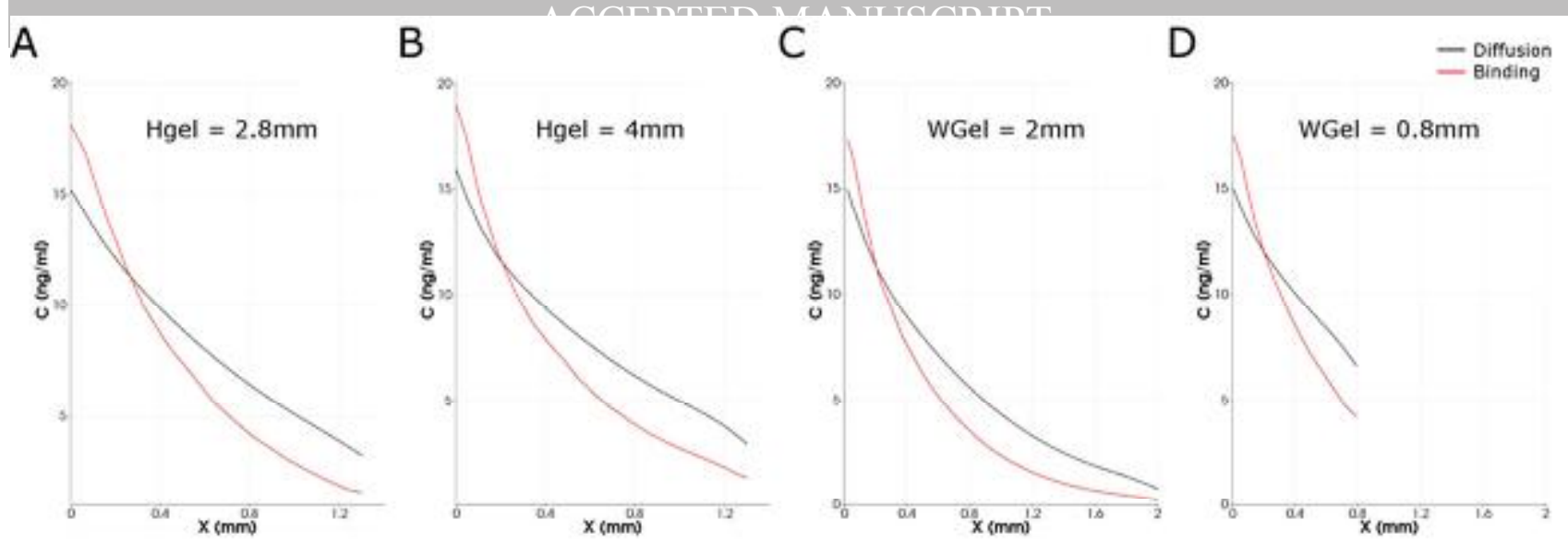

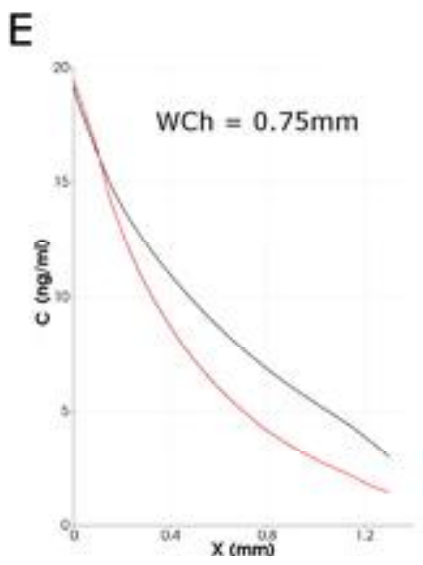

$\mathrm{F}$

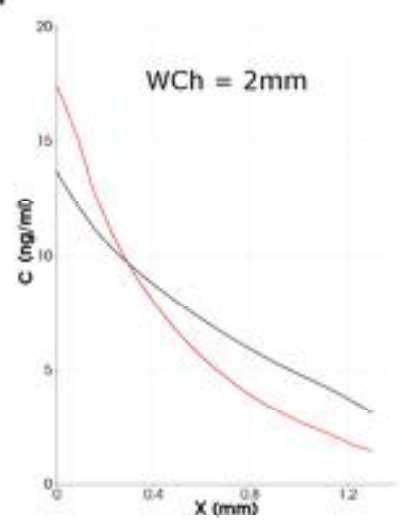

I

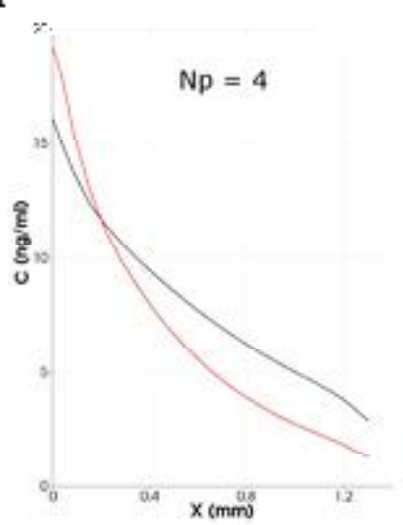

]

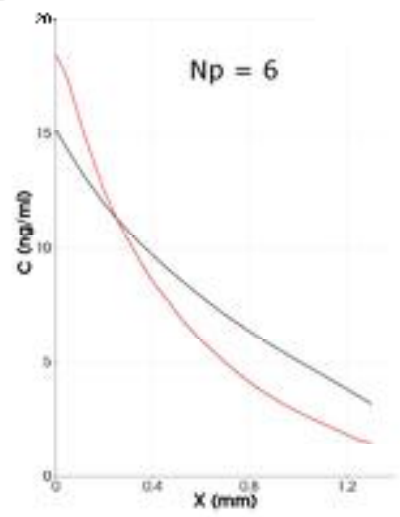

G

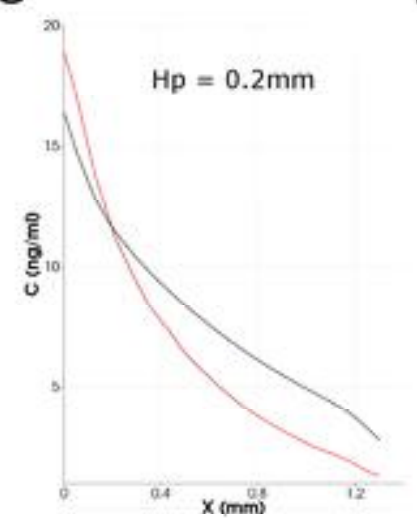

$\mathrm{K}$

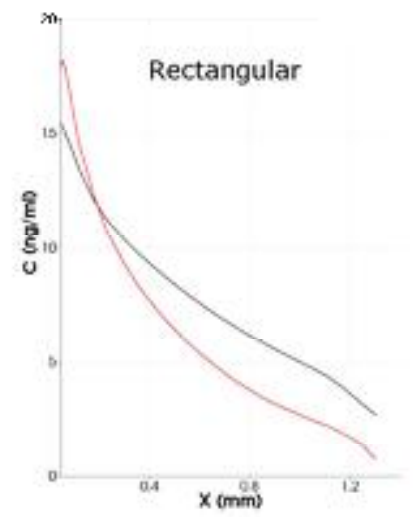

$\mathrm{H}$

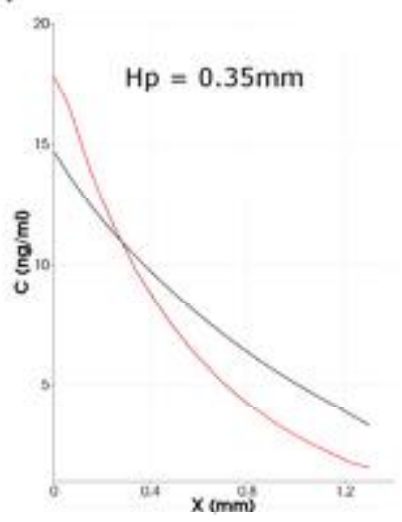

$\mathrm{L}$

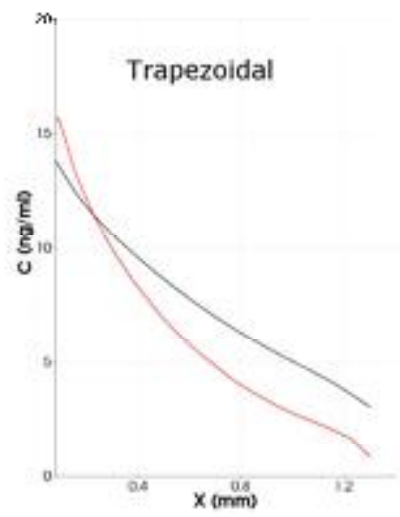

Figure 6. Parametric analysis on chip-1 device. Numerical predictions of diffusion and binding profiles after 24 hours of diffusion of PDGF-BB within a collagen type I hydrogel with: $H_{g e l}=3.2 \mathrm{~mm}, W_{g e l}=1.3 \mathrm{~mm}, W_{c h}=1 \mathrm{~mm}, H_{p}=0.3 \mathrm{~mm}, N_{p}=5$ (the results are showed in Fig. 5A). Each panel shows the obtained results by varying the specified parameter A) $H_{g e l}=2.8 \mathrm{~mm}$, B) $H_{g e l}=4 \mathrm{~mm}$, C) $W_{g e l}=2 \mathrm{~mm}$, D) $W_{g e l}=0.8 \mathrm{~mm}$, E) $W_{c h}=0.75 \mathrm{~mm}$, F) $\left.\left.W_{c h}=2 \mathrm{~mm}, \mathrm{G}\right) H_{p}=0.2 \mathrm{~mm}, \mathrm{H}\right) H_{p}=0.35 \mathrm{~mm}$, I) $N_{p}=4, \mathrm{~J}$ ) $\left.N_{p}=6, K\right)$ rectangular and L) trapezoidal type posts. 


\section{Numerical predictions of GF transport under different hydrogel combinations}

Having explored the GF distribution within different microfluidic devices, the effect of the combination of different hydrogels inside a microfluidic device was studied. To that end, GF diffusion under two different hydrogel configurations within the chip-4 (the double channel device) was studied: collagen-fibrin and fibrincollagen (see Fig. 7). The simulated cases are the diffusion of PDGF-BB and TGF- $\beta_{1}$ growth factor between collagen and fibrin adjacent hydrogels under different configurations, and the total time simulated was 24 hours. The parameter values that define chip-4 device geometry are: $H_{\text {gel }}=3 \mathrm{~mm}, W_{\text {gel } 1,2}=1.5 \mathrm{~mm}, W_{c h}=0.9 \mathrm{~mm}$, $H_{p}=0.25 \mathrm{~mm}, N_{p}=5$ and with rectangular posts geometry.

To determine whether there were significate differences between diffusion gradients for the different simulated cases, the same initial concentration of GF was applied for each case $(50 \mathrm{ng} / \mathrm{ml})$ and the total time simulated was 24 hours for all cases. The radius of TGF- $\beta_{1}$ particle was estimated from Protein Data Bank to be $3.810^{-9} \mathrm{~m}$, which according to equation 3 corresponds to a standard diffusion coefficient of $1.4910^{-11} \mathrm{~m}^{2} / \mathrm{s}$. The fibrin hydrogel characterization was previously performed with confocal reflection imaging and fibrin fiber radius was estimated to be $66.53 \pm 13.57 \mathrm{~nm}$ (mean $\pm \mathrm{se}$ ) and the fibrin void ratio to be $71.46 \pm 1 \%$ (mean $\pm \mathrm{se}$ ). The standard and effective coefficients of diffusion of PDGF-BB and TGF- $\beta_{1}$ in collagen and fibrin as well as the degradation and binding rates used in all simulations (experimentally adjusted, [14]) may be consulted in Table 2 .

\section{Table 2}

Main parameters used in the simulations, where $c$ and $f$ refers to collagen and fibrin hydrogels, respectively.

\begin{tabular}{clcc}
\hline Parameter & \multicolumn{1}{c}{ Description } & PDGF-BB & TGF- $\boldsymbol{\beta}$ 1 \\
\hline$D_{\infty}$ & Standard diffusion coefficient & $1.2610^{-11} \mathrm{~m}^{2} / \mathrm{s}$ & $1.4910^{-11} \mathrm{~m}^{2} / \mathrm{s}$ \\
$D_{c}$ & Effective diffusion of a GF in collagen & $0.7710^{-11} \mathrm{~m}^{2} / \mathrm{s}$ & $0.9210^{-11} \mathrm{~m}^{2} / \mathrm{s}$ \\
$D_{f}$ & Effective diffusion of a GF in fibrin & $0.7010^{-11} \mathrm{~m}^{2} / \mathrm{s}$ & $0.83 \mathrm{e}^{-11} \mathrm{~m}^{2} / \mathrm{s}$ \\
$K_{d, c}$ & Degradation rate in collagen & $310^{-6} \mathrm{~s}^{-1}$ & $510^{-6} \mathrm{~s}^{-1}$ \\
$K_{d, f}$ & Degradation rate in fibrin & $310^{-6} \mathrm{~s}^{-1}$ & $4.510^{-6} \mathrm{~s}^{-1}$ \\
$K_{b, c}$ & Binding rate in collagen & $110^{-5} \mathrm{~s}^{-1}$ & $110^{-5} \mathrm{~s}^{-1}$ \\
$K_{b, f}$ & Binding rate in fibrin & $110^{-7} \mathrm{~s}^{-1}$ & $210^{-5} \mathrm{~s}^{-1}$ \\
\hline
\end{tabular}

Fig. 7 shows diffusion profiles generated in the central domain of the device for the different studied cases. For both diffusion cases it can be found a slope change on the diffusion profiles as a consequence of a hydrogel domain change, which can be explained by the differences in the structural characteristics of each hydrogel, such as the network concentration (pore size) or the fibril diameter. Moreover, the changes on the final concentration values obtained after 24 hours of GF diffusion simulation of PDGF-BB and TGF- $\beta_{1}$ can be mainly explained by the differences on the particle size and on the binding rates of each factor to the hydrogel. 


\section{A}
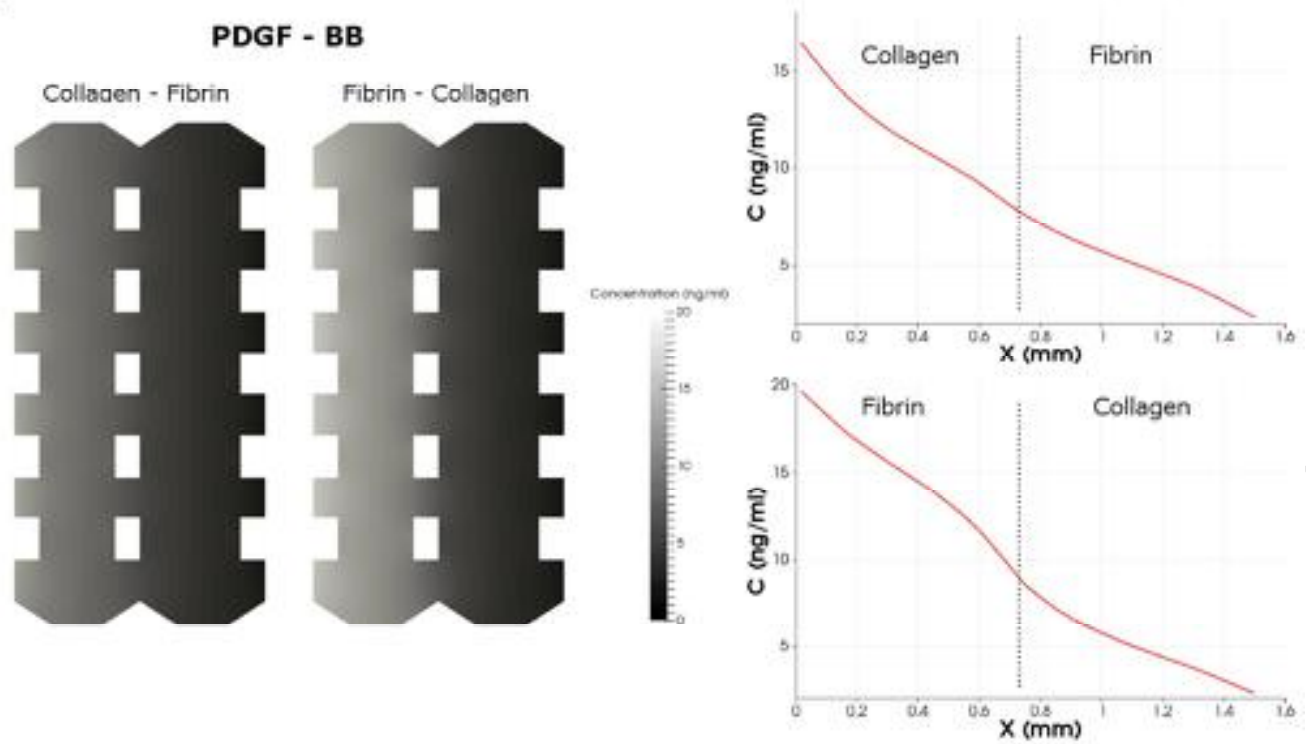

B

TGF $-\boldsymbol{\beta}_{1}$
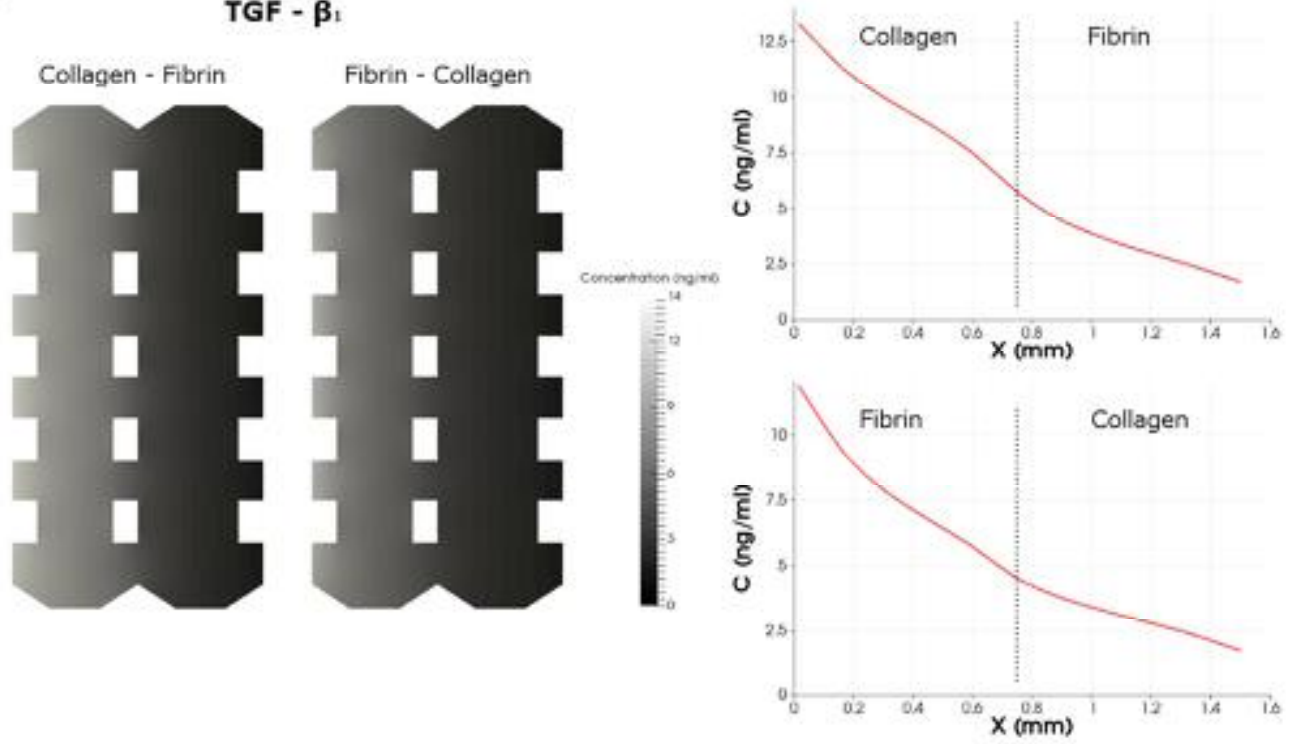

Figure 7. Effect of different hydrogel combination on the diffusion profiles generated inside a microfluidic device. Numerical predictions of the spatial distribution and diffusion profiles obtained after 24 hours of diffusion of: A) PDGFBB and B) TGF- $\beta_{1}$, in collagen-fibrin and fibrin-collagen hydrogel configurations within chip-4 device.

Taken together, these results remark the importance to have a better knowledge on the behavior of a GF's within an extracellular matrix, more specially, in the interface between two different matrices or hydrogels to better understand relevant physiological processes such as wound healing processes. Actually, by this step-gradient hydrogel, we can recreate in-vitro how cells behave under a strong alteration of the extracellular matrix guided by different chemical gradients [33]. Interestingly, these two-chambers microfluidic devices allowed us to analyze several cell functions through the interface of both materials and under different chemical gradients, including migration, proliferation, and matrix remodeling, in real time. 


\section{Automatic quantification of growth factor signaling: a web application}

To facilitate the quantification of the chemical gradients generated within a synthetic hydrogel inside a microfluidic-based chip, an automatic quantification through a web application is presented. As proposed in materials and methods section, this application allows any user to automatically design a microfluidic device, starting from four different base models of microfluidic devices. Moreover, the user may choose the input parameters that define the diffusion case to simulate. The main objective of this application is to facilitate to the scientific community the characterization of gradient formation processes within a synthetic hydrogel and therefore reduce device design times and facilitate the experimental validation and parameter selection processes. To ensure the day-to-day applicability, user requirements were investigated prior to tool development (parametrical and sensibility study was performed) so that the 4 designs presented and their possible variations cover a wide range of common necessities. The tool may be consulted on http://m2be.unizar.es/insilico cell under the software tab, and freely used by any user after registration. The use of this software is restricted to site members. Therefore, if you are not an existing user, you may register as a new user by filling the required fields.

Figure 8 shows the panel seen by the user after parameter selection (left) and the results returned to the website after the simulation (right). Chip-1 device was selected as example with the following geometric parameters of: $H_{g e l}=3.2 \mathrm{~mm}, W_{g e l}=1.3 \mathrm{~mm}, W_{c h}=0.8 \mathrm{~mm}, H_{p}=0.3 \mathrm{~mm}, N_{p}=5$ and with trapezoidal posts geometry. The diffusion case simulated was the PDGF-BB diffusion within a collagen type I hydrogel during 24 hours and with an initial concentration of the growth factor equal to $50 \mathrm{ng} / \mathrm{ml}$.

Chip 1
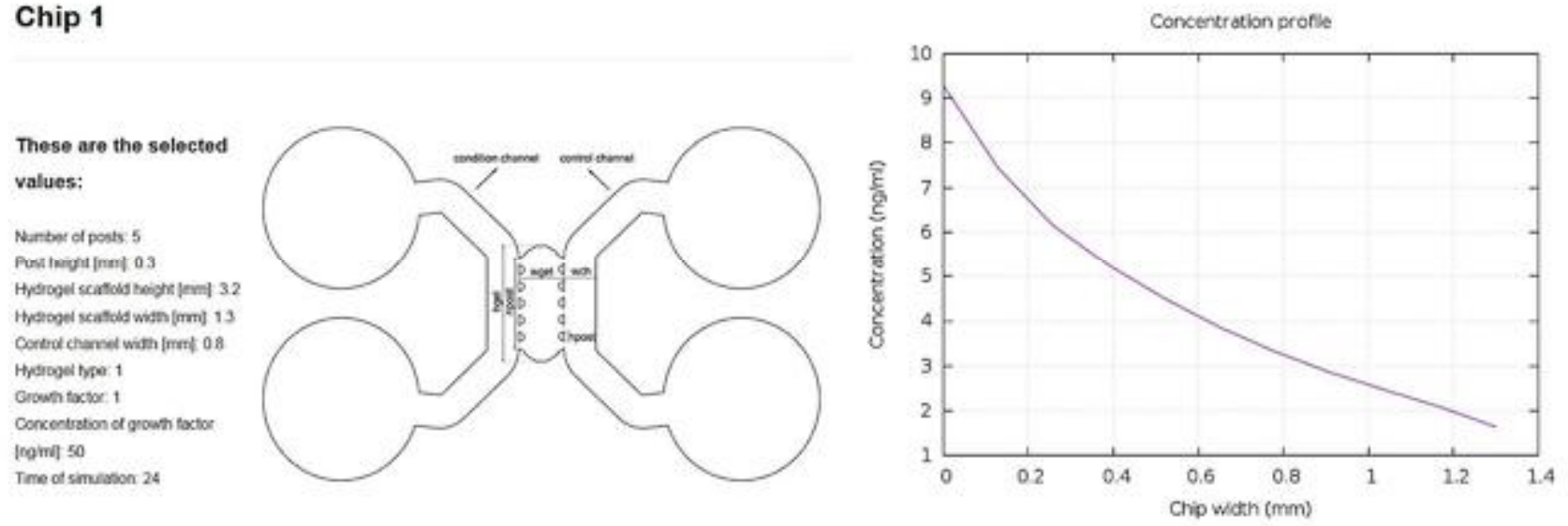

Figure 8. Web application screenshot. Left panel shows the set of user-selected parameters as well as a scheme of the chosen chip. Right panel shows the concentration/diffusion profile generated within the hydrogel domain returned to the website, indicating the microfluidic device as well as the calculation parameters selected.

\section{Discussion}

Due to the importance that microfluidic technology has acquired during the last few years on in-vitro 3-D cell cultures, multiple systems have been developed to study the mobility of cells under different controlled conditions, such as fluid flow and chemoattractant or chemorepellent molecules [33, 39, 40]. Thus, it is crucial to deeply study the spatio-temporal distribution of the chemical substances within a synthetic hydrogel to better understand the cell response under different signaling pathways.

The finite element model here presented is based on a reaction-diffusion transport model and allows an automatic prediction of the chemical gradients generated inside a microfluidic device for any growth factor 
or chemical substance for a wide range of geometries. Furthermore, a web-based application is developed to automatically calculate the spatio-temporal distribution of a growth factor, across hydrogel-based matrices (specifically collagen type I and fibrin) bringing to any user the opportunity to explore different geometries and conditions before investing on real experiments. In fact, this application yields an accurate prediction, based on previous experimental results, where diffusion phenomenon can be estimated within a microdevice. To that end, quantitative ELISA-assays for growth factor concentrations measurement were previously performed and the behavior of die dextran diffusion gradient was studied, validating therefore the numerical model proposed [14].

Firstly, four different microfluidic device geometries were defined to study the dependence of the geometrical parameters onto the gradient formation processes. To that end, and to notably decrease the computational time, a simplification of the device geometry was performed (disregarding the deposits) and assuming that the chemical factor diffuses homogeneously throughout the condition channel of the device and the initial growth factor concentration is constant in both lateral channels. The numerical predictions obtained after diffusion of $50 \mathrm{ng} / \mathrm{ml}$ of PDGF-BB within a collagen gels for the four different models of microfluidic devices, showed differences on the concentration profiles obtained for each case.

Thus, to understand how the different parameters that characterize these microfluidic devices influence the behavior of the diffusion processes in 3D, an intensive multiparametric analysis was performed. As expected, the results demonstrate that growth factor diffusion within 3-D matrices clearly depends not only on the physics of diffusion, but also on the geometrical parameters that characterizes these complex devices. Consequently, and after studying the diffusion and binding profiles generated for the different cases, we found a stronger impact on the gradient formation process for the following parameters: the hydrogel scaffold height and width $\left(H_{g e l}\right.$ and $\left.W_{g e l}\right)$, the lateral channels width $\left(W_{c h}\right)$, the post height $\left(H_{p}\right)$ and the number of posts $\left(N_{p}\right)$. Differences on concentration for condition and control channels with respect to the reference case (chip-1 with the reference parameter values) were quantified. For instance, changes on hydrogel width generated differences of up to $21 \%$ on condition channel concentration and of about $24 \%$ on control channel concentrations; and changes on post height showed differences of $6 \%$ on condition channel concentration and of $13 \%$ on control channel concentrations.

Additionally, the effect of the combination of different hydrogels inside a microfluidic device was studied. In particular, PDGF-BB and TGF- $\beta_{1}$ diffusion under two different hydrogel configurations within the chip-4 was studied: collagen-fibrin and fibrin-collagen. Results showed differences on the slope of the diffusion profiles generated in the central domain of the device for the different cases of study, then highlighting the importance of studying the combination of different matrices or hydrogels on physiologically relevant microenvironments.

To understand the spatial distribution of the complex chemical gradient generated by diffusion into the hydrogel scaffold, two-dimensional finite element simulations have been previously presented by using commercial software's, such as COMSOL or ANSYS $[41,42]$. However, the commercial software's do not offer the possibility to determine the concentration gradients as a function of a reactive term that incorporates additional phenomena, such as the degradation and the binding of the factor to the fibrillar network.

In sum, the novel web application here presented facilitates to any user an automatic estimation of growth factor diffusion within 3-dimensional matrices inside a microfluidic device without prior knowledge of CAD or Finite element modeling. This application allows the user to design his/her own device by defining online the main geometrical parameters that characterize these devices (hydrogel height and width, lateral channels width, post height and number of posts); as well as the input parameters that define the diffusion case: the growth factor to diffuse (PDGF-BB and TGF- $\beta 1$ ), the initial growth factor concentration, the type of matrix (collagen of fibrin) and the diffusion time to simulate. Thus, the automatization of microfluidic device geometries generation provide a powerful tool which facilitates to any user the possibility to automatically create its own microfluidic device, greatly reducing the experimental validation processes and advancing in 


\section{ACCEPTED MANUSCRIPT}

the understanding of in-vitro 3-D cell responses without the necessity of using commercial software or performing real testing experiments.

\section{Acknowledgments}

The project was supported by the European Research Council (ERC-2012-StG 306571 JMGA), the Spanish Ministry of Economy and Competitiveness (DPI2015-64221-C2-1-R) and the Government of Aragon (C126/2015 MC).

\section{Conflict of interest statement}

The authors declare that they have no conflict of interest.

\section{References}

1. Li, L., et al., Collective cell migration: Implications for wound healing and cancer invasion. Burns \& trauma, 2013. 1(1): p. 21.

2. Martin, P., Wound healing - Aiming for perfect skin regeneration. Science, 1997. 276(5309): p. 75-81.

3. Friedl, P. and D. Gilmour, Collective cell migration in morphogenesis, regeneration and cancer. Nature Reviews Molecular Cell Biology, 2009. 10(7): p. 445-457.

4. Wang, W.G., et al., Tumor cells caught in the act of invading: their strategy for enhanced cell motility. Trends in Cell Biology, 2005. 15(3): p. 138-145.

5. Garrett, Q., et al., Involvement of CTGF in TGF-81-Stimulation of Myofibroblast Differentiation and Collagen Matrix Contraction in the Presence of Mechanical Stress. Investigative Ophthalmology \& Visual Science, 2004. 45(4): p. 1109-1116.

6. Long, C.J., et al., Fibroblast growth factor-2 promotes keratan sulfate proteoglycan expression by keratocytes in vitro. Journal of Biological Chemistry, 2000. 275(18): p. 13918-13923.

7. Jester, J.V. and J. Ho-Chang, Modulation of cultured corneal keratocyte phenotype by growth factors/cytokines control in vitro contractility and extracellular matrix contraction. Exp Eye Res, 2003. 77(5): p. 581-92.

8. Roussos, E.T., J.S. Condeelis, and A. Patsialou, Chemotaxis in cancer. Nat Rev Cancer, 2011. 11(8): p. 573-87.

9. Petrie, R.J., A.D. Doyle, and K.M. Yamada, Random versus directionally persistent cell migration. Nat Rev Mol Cell Biol, 2009. 10(8): p. 538-49.

10. Aznavoorian, S., et al., Signal transduction for chemotaxis and haptotaxis by matrix molecules in tumor cells. J Cell Biol, 1990. 110(4): p. 1427-38.

11. Kramer, N., et al., In vitro cell migration and invasion assays. Mutation Research-Reviews in Mutation Research, 2013. 752(1): p. 10-24.

12. Zervantonakis, I.K., et al., Three-dimensional microfluidic model for tumor cell intravasation and endothelial barrier function. Proceedings of the National Academy of Sciences, 2012. 109(34): p. 13515-13520.

13. Lee, J.H., et al., Microfluidic 3D bone tissue model for high-throughput evaluation of wound-healing and infection-preventing biomaterials. Biomaterials, 2012. 33(4): p. 999-1006.

14. Moreno-Arotzena, O., et al., Inducing chemotactic and haptotactic cues in microfluidic devices for three-dimensional in vitro assays. Biomicrofluidics, 2014. 8(6).

15. Wu, J.D., X. Wu, and F. Lin, Recent developments in microfluidics-based chemotaxis studies. Lab on a Chip, 2013. 13(13): p. 2484-2499.

16. Kothapalli, C.R., et al., A high-throughput microfluidic assay to study neurite response to growth factor gradients. Lab Chip, 2011. 11(3): p. 497-507.

17. Taipale, J. and J. Keski-Oja, Growth factors in the extracellular matrix. Faseb Journal, 1997. 11(1): $\mathrm{p}$. 51-9. 
18. Brizzi, M.F., G. Tarone, and P. Defilippi, Extracellular matrix, integrins, and growth factors as tailors of the stem cell niche. Current Opinion in Cell Biology, 2012. 24(5): p. 645-51.

19. Martino, M.M., et al., Growth Factors Engineered for Super-Affinity to the Extracellular Matrix Enhance Tissue Healing. Science, 2014. 343(6173): p. 885-888.

20. Peppas, N.A., et al., Hydrogels in biology and medicine: from molecular principles to bionanotechnology. Advanced Materials, 2006. 18(11): p. 1345-1360.

21. Geckil, H., et al., Engineering hydrogels as extracellular matrix mimics. Nanomedicine, 2010. 5(3): p. 469-484.

22. Frantz, C., K.M. Stewart, and V.M. Weaver, The extracellular matrix at a glance. J Cell Sci, 2010. 123(Pt 24): p. 4195-200.

23. Diegelmann, R.F. and M.C. Evans, Wound healing: An overview of acute, fibrotic and delayed healing. Frontiers in Bioscience, 2004. 9: p. 283-289.

24. Weisel, J.W., The mechanical properties of fibrin for basic scientists and clinicians. Biophysical Chemistry, 2004. 112(2-3): p. 267-276.

25. Ern, A. and J.-L. Guermond, Theory and practice of finite elements. 2010, New York: Springer.

26. Martino, M.M., et al., Heparin-binding domain of fibrin (ogen) binds growth factors and promotes tissue repair when incorporated within a synthetic matrix. Proceedings of the National Academy of Sciences, 2013. 110(12): p. 4563-4568.

27. Kihara, T., J. Ito, and J. Miyake, Measurement of biomolecular diffusion in extracellular matrix condensed by fibroblasts using fluorescence correlation spectroscopy. PLoS One, 2013. 8(11): p. e82382.

28. Einstein, A., Über die von der molekularkinetischen Theorie der Wärme geforderte Bewegung von in ruhenden Flüssigkeiten suspendierten Teilchen. Annalen der physik, 1905. 322(8): p. 549-560.

29. Ogston, A., B. Preston, and J. Wells, On the transport of compact particles through solutions of chainpolymers. Proceedings of the Royal Society of London. A. Mathematical and Physical Sciences, 1973. 333(1594): p. 297-316.

30. Kim, W.-S. and J.M. Tarbell, Macromolecular transport through the deformable porous media of an artery wall. Journal of biomechanical engineering, 1994. 116(2): p. 156-163.

31. Moreno-Arotzena, O., et al., Characterization of Fibrin and Collagen Gels for Engineering Wound Healing Models. Materials (Basel), 2015. 8(4): p. 1636-1651.

32. Del Amo, C., et al., Quantification of angiogenic sprouting under different growth factors in a microfluidic platform. Journal of Biomechanics, 2016. 49(8): p. 1340-1346.

33. Del Amo, C., et al., Quantifying 3D chemotaxis in microfluidic-based chips with step gradients of collagen hydrogel concentrations. Integrative Biology, 2017. 9(4): p. 339-349.

34. Li, J., et al., Microfluidic device for studying cell migration in single or co-existing chemical gradients and electric fields. Biomicrofluidics, 2012. 6(2): p. 24121-2412113.

35. Geuzaine, C. and J.F. Remacle, Gmsh: A 3-D finite element mesh generator with built-in pre- and post-processing facilities. International Journal for Numerical Methods in Engineering, 2009. 79(11): p. 1309-1331.

36. Rüberg, T., F. Cirak, and J.M.G. Aznar, An unstructured immersed finite element method for nonlinear solid mechanics. Advanced Modeling and Simulation in Engineering Sciences, 2016. 3(1): p. 22.

37. Ern, A. and J.-L. Guermond, Theory and practice of finite elements. Vol. 159. 2013: Springer Science \& Business Media.

38. Thain, D., T. Tannenbaum, and M. Livny, Distributed computing in practice: the Condor experience. Concurrency and Computation-Practice \& Experience, 2005. 17(2-4): p. 323-356.

39. Wang, S.J., et al., Differential effects of EGF gradient profiles on MDA-MB-231 breast cancer cell chemotaxis. Experimental Cell Research, 2004. 300(1): p. 180-189.

40. Hughes-Alford, S.K. and D.A. Lauffenburger, Quantitative analysis of gradient sensing: towards building predictive models of chemotaxis in cancer. Current Opinion in Cell Biology, 2012. 24(2): p. 284-291.

41. Sudo, R., et al., Transport-mediated angiogenesis in 3D epithelial coculture. Faseb Journal, 2009. 23(7): p. 2155-2164. 
ACCEPTED MANUSCRIPT

42. Jeong, G.S., et al., Sprouting Angiogenesis under a Chemical Gradient Regulated by Interactions with an Endothelial Monolayer in a Microfluidic Platform. Analytical Chemistry, 2011. 83(22): p. 84548459. 
The main highlights of this work are the following:

- We present a novel web-based application which facilitates an automatic estimation of growth factor diffusion through 3-dimensional matrices inside a microfluidic device.

- The tool allows defining the geometrical parameters that characterize a microfluidic chip design as well as the diffusion-related parameters, enabling to any user the possibility to customize its own microfluidic device.

- Simulations are performed remotely and automatically, returning the results to the web page in less than 3 minutes.

- The application facilitates the scientific community the characterization of gradient formation processes within a synthetic hydrogel, reducing device design times and experimental validation and parameter selection processes. 